Ermelinda De Meo, MD

Lucia Moiola, MD

Angelo Ghezzi, MD

Pierangelo Veggiotti, MD

Ruggero Capra, MD

Maria Pia Amato, MD

Elisabetta Pagani, MSc

Agnese Fiorino, PhD

Lorena Pippolo, MD

Maria C. Pera, MD

Giancarlo Comi, MD

Andrea Falini, MD

Massimo Filippi, MD

Maria A. Rocca, MD

Correspondence to

Dr. Rocca:

rocca.mara@hsr.it
Supplemental data at Neurology.org

\section{MRI substrates of sustained attention system and cognitive impairment in pediatric MS patients}

ABSTRACT

Objective: To explore the structural and functional integrity of the sustained attention system in patients with pediatric multiple sclerosis (MS) and its effect on cognitive impairment.

Methods: We enrolled 57 patients with pediatric MS and 14 age- and sex-matched healthy controls (HCs). Patients with >3 abnormal tests at neuropsychological evaluation were classified as cognitively impaired (CI). Sustained attention system activity was studied with fMRI during the Conners Continuous Performance Test (CCPT). Structural integrity of attention network connections was quantified with diffusion tensor (DT) MRI.

Results: Within-group analysis showed similar patterns of recruitment of the attention network in $\mathrm{HCs}$ and patients with pediatric MS. Diffuse network DT MRI structural abnormalities were found in patients with MS. During CCPT, with increasing task demand, patients with pediatric MS showed increased activation of the left thalamus, anterior insula, and anterior cingulate cortex (ACC) and decreased recruitment of the right precuneus compared to HCs. Thirteen patients (23\%) were classified as $\mathrm{Cl}$. Compared to cognitively preserved patients, $\mathrm{Cl}$ patients with pediatric MS had decreased recruitment of several areas located mainly in parietal and occipital lobes and cerebellum and increased deactivation of the ACC, combined with more severe structural damage of white matter tracts connecting these regions.

Conclusions: Our results suggest that the age-expected level of sustained attention system functional competence is achieved in patients with pediatric MS. Inefficient regulation of the functional interaction between different areas of this system, due to abnormal white matter integrity, may result in global cognitive impairment in these patients. Neurology ${ }^{\circledR}$ 2017;89:1265-1273

\section{GLOSSARY}

ACC $=$ anterior cingulate cortex CCPT $=$ Conners Continuous Performance Test; $\mathbf{C l}=$ cognitively impaired; $\mathbf{C P}=$ cognitively preserved; $\mathbf{D M N}=$ default mode network; $\mathbf{D T}=$ diffusion tensor; $\mathbf{F A}=$ fractional anisotropy; $\mathbf{F C}=$ functional connectivity; HC = healthy control; $\mathbf{M S}$ = multiple sclerosis; $\mathbf{R S}=$ resting state; $\mathbf{S F G}=$ superior frontal gyrus; $\mathbf{W M}$ = white matter.

Sustained attention represents a key executive function underlying higher attentional processes (divided and selective attention) and global cognitive functioning, ${ }^{1}$ the functional maturation of which occurs during late childhood and adolescence, as demonstrated by $\mathrm{fMRI}^{2}$ and $\mathrm{EEG}^{3,4}$ investigations. In this perspective, the onset of multiple sclerosis (MS) in this critical age may have important and distinct consequences for cognitive abilities.

To evaluate sustained attention and cognitive control capabilities and their maturation during the developmental age, the Conners Continuous Performance Test (CCPT) has been frequently used. ${ }^{5}$ Using fMRI during a sustained attention task, ${ }^{6}$ a previous study investigated brain functional changes between childhood and adulthood, describing increased activation with development in fronto-temporo-parieto-cerebellar regions that mediate sustained attention, confirming a continued functional development of these regions throughout childhood to middle adulthood.

From the Neuroimaging Research Unit (E.D.M., E.P., M.F., M.A.R.) and Department of Neurology (E.D.M., L.M., A. Fiorino, G.C., M.F., M.A.R.), Institute of Experimental Neurology, Division of Neuroscience, and Department of Neuroradiology (A. Falini), San Raffaele Scientific Institute, Vita-Salute San Raffaele University, Milan; Multiple Sclerosis Center (A.G., L.P.), Ospedale di Gallarate; Department of Child Neurology and Psychiatry (P.V., M.C.P.), C. Mondino National Neurological Institute, Brain and Behaviour Department, University of Pavia; Multiple Sclerosis Center (R.C.), Spedali Civili of Brescia; and Department NEUROFARBA (M.P.A.), Section Neurosciences, University of Florence, Italy. Go to Neurology.org for full disclosures. Funding information and disclosures deemed relevant by the authors, if any, are provided at the end of the article. 
Recent preliminary evidence in patients with pediatric MS has resulted in the hypothesis that the disease may influence the maturation of brain structures. Using volumetric MRI, a longitudinal study showed a failure of age-expected brain growth in these patients. ${ }^{7}$ A diffusion tensor (DT) MRI study found impaired maturation of white matter (WM) tracts in patients with very early-onset disease. $^{8}$

By applying an active fMRI paradigm aimed at testing sustained attention and executive functions, this study investigates the recruitment of the sustained attention system with increasing task demand in patients with pediatric MS compared to age- and sexmatched healthy controls (HCs) to assess whether and how MS onset during childhood compromises this functional network. Starting from the consideration that abnormalities in sustained attention are frequently associated with behavioral, learning, emotional, and cognitive difficulties in adolescence ${ }^{9}$ and that attention is one of the most frequent areas of impairment in patients with pediatric MS, ${ }^{10}$ we investigated the relationship between cognitive impairment and sustained attention system recruitment abnormalities in these patients. To clarify the role of network structural abnormalities on fMRI findings, we also quantified the structural integrity of the connections between brain regions relevant to the task using DT MRI.

METHODS Ethics committee approval. Approval was received from the local ethical standards committee on human experimentation, and written informed consent was obtained from all participants and their parents before study enrolment.

Participants. We enrolled 57 consecutive, right-handed pediatric patients with relapsing-remitting MS referred to specialized centers for the diagnosis of pediatric MS. Inclusion and exclusion criteria are reported in appendix e-1 at Neurology.org. Fourteen sex- and age-matched HCs with no history of neurologic dysfunction and a normal neurologic examination served as the control group.

Clinical and neuropsychological assessment. All patients underwent a neurologic examination with rating on the Expanded Disability Status Scale ${ }^{11}$ and a neuropsychological assessment with a Neuropsychological Battery for Children, standardized and validated for an Italian pediatric population with MS (appendix e-1). ${ }^{12,13}$ Global premorbid cognitive functioning with IQ was assessed through the Wechsler Intelligence Scale for Children. ${ }^{14}$ Patients with an abnormal performance in $\geq 3$ tests were classified as cognitively impaired (CI). ${ }^{12}$ As previously described, ${ }^{15} z$ scores (based on a population of pediatric HCs matched for age and education) ${ }^{16}$ for each of the previous domains and a global $z$ score of cognitive function (obtained by averaging $z$ scores of all tests) were calculated.

fMRI experimental design. The computerized version of the $\mathrm{CCPT}^{17}$ was implemented with the Presentation software (www.neuro-bs.com, version 14.8), as described in detail in appendix e-1. All participants were trained to perform the task before MRI acquisition. Percentages of correct and incorrect responses and reaction times were recorded. All participants completed the fMRI acquisition without interruption.

MRI acquisition and analysis. Appendix e-1 provides a detailed description of the MRI acquisition and analysis protocol. From all participants, the following sequences were obtained: T2*-weighted single-shot echo-planar imaging scan during the CCPT task (SPM12), DT MRI scan, dual-echo turbo spin-echo scan, and 3-dimensional T1-weighted fast-field-echo scan. T2hyperintense and T1-hypointense lesion volumes were measured on the dual-echo turbo spin-echo and 3-dimensional T1weighted scans with a local thresholding segmentation technique (Jim 6, Xinapse Systems, West Bergholt, UK). Normalized brain volume, WM volume, and gray matter volume were measured on the 3-dimensional T1-weighted scans with SIENAx software after T1-hypointense lesion refilling. ${ }^{18}$

Analysis of DTI data (FSL software) focused on tracts connecting brain regions identified by fMRI analysis as key regions involved in CCPT performance with an approach similar to that applied by previous authors. ${ }^{19,20}$ Tracts were generated between 3 -mm-radius regions of interest on the basis of the peak activation or deactivation during the CCPT from HCs. Fourteen regions of interest were used, and all possible combinations between them were explored. To overcome the problems associated with probabilistic tractography due to WM lesions, tract probability maps were obtained from the $\mathrm{HC}$ group and then back-projected in individual space to obtain a mean fractional anisotropy (FA) value per tract for all the study participants. ${ }^{21}$

Statistical analysis. Between-group comparisons of clinical, demographic, and structural MRI parameters were performed with the Pearson $\chi^{2}$ test, Mann-Whitney $U$ test, or KruskalWallis test adjusted for age and sex and corrected for multiple comparisons, as appropriate. CCPT performance was compared between groups with an analysis of variance, adjusted for age and sex.

A second-level analysis with SPM12 was performed to assess the average fMRI activation and deactivation during the CCPT (i.e., average activation of the ISI-1, ISI-2, and ISI-4 conditions) and the load effect in HCs and patients with pediatric MS (as a whole and according to the presence/absence of cognitive impairment) (1-sample $t$ test); the differences in fMRI activation between study groups (2-sample $t$ test and full factorial models, age and sex adjusted); and the correlation between fMRI activity during the load condition and behavioral (accuracy, reaction time), clinical (Expanded Disability Status Scale, disease duration), neuropsychological (global $z$ score of cognitive performance and $z$ scores of single cognitive domains, including attention), and structural MRI (T2 lesion volume, atrophy, and FA measures) variables (multiple regression models, adjusted for age and sex; 1 separate model for each variable).

To assess the fMRI abnormalities in a given patient group vs the others included in the full factorial models, we performed a conjunction analysis, ${ }^{22}$ which, by testing for the conjunction of different hypotheses (each described as 


\begin{tabular}{|c|c|c|c|c|c|c|}
\hline \multirow[t]{2}{*}{ Table 1} & \multirow[b]{2}{*}{$\mathrm{HCs}$} & \multirow{3}{*}{$\begin{array}{l}\text { Patients with } \\
\text { pediatric MS } \\
57\end{array}$} & \multirow{3}{*}{$\begin{array}{l}p \text { Values }^{a} \\
-\end{array}$} & \multirow{3}{*}{$\begin{array}{l}\text { CP patients } \\
\text { with MS } \\
44\end{array}$} & \multirow{3}{*}{$\begin{array}{l}\mathrm{Cl} \text { patients } \\
\text { with MS } \\
13\end{array}$} & \multirow{3}{*}{$\begin{array}{l}\text { p Values } \\
- \\
-\end{array}$} \\
\hline & & & & & & \\
\hline Participants, $n$ & 14 & & & & & \\
\hline Girls/boys, $n$ & $8 / 6$ & $36 / 21$ & $0.17^{b}$ & $30 / 14$ & $6 / 7$ & $0.40^{\mathrm{b}}$ \\
\hline Mean age (range), y & $13.6(8.8-17.9)$ & $15.0(7.6-18.0)$ & 0.12 & $15.2(11.1-18.0)$ & $15.1(7.6-17.7)$ & 0.58 \\
\hline Median EDSS (range) & - & $1.0(0.0-4.0)$ & - & $1.0(0.0-4.0)$ & $1.5(0.0-4.0)$ & 0.37 \\
\hline Median disease duration (range), y & - & $1.7(0.1-8.1)$ & - & $1.4(0.1-6.8)$ & $3.5(0.2-8.1)$ & 0.03 \\
\hline Mean T2 LV (SD), mL & - & $6.0(7.8)$ & - & $4.2(5.2)$ & $9.6(11.6)$ & 0.26 \\
\hline Mean T1 LV (SD), mL & - & $3.7(5.0)$ & - & $2.6(3.0)$ & $6.5(8.6)$ & 0.31 \\
\hline Mean NBV (SD), mL & $1728(74)$ & $1660(79)$ & 0.01 & $1674(68)$ & $1612(88)$ & 0.03 \\
\hline Mean GMV (SD), mL & $882(67)$ & $830(55)$ & 0.02 & $835(55)$ & $808(59)$ & 0.12 \\
\hline Mean WMV (SD), mL & $846(37)$ & $830(48)$ & 0.43 & $839(38)$ & $803(46)$ & 0.01 \\
\hline Mean CCPT correct responses (range), \% & $95(82-100)$ & $93(84-100)$ & 0.21 & $94(88-100)$ & $93(87-98)$ & 0.04 \\
\hline Mean CCPT reaction time (SD), ms & $0.4(0.1)$ & $0.4(0.1)$ & 0.55 & $0.4(0.6)$ & $0.4(0.7)$ & 0.41 \\
\hline
\end{tabular}

Abbreviations: $\mathrm{CCPT}=$ Conners Continuous Performance Test; $\mathrm{Cl}=$ cognitively impaired; $\mathrm{CP}=$ cognitively preserved; EDSS = Expanded Disability Status Scale; GMV = gray matter volume; HC = healthy control; LV = lesion volume; MS = multiple sclerosis; $\mathrm{NBV}=$ normalized brain volume; WMV = white matter volume.

${ }^{a}$ Mann Whitney $U$ test.

${ }^{b}$ Chi-square test.

a contrast), allows us to identify where several fMRI clusters were jointly significant by evaluating the significance of combined contrasts.

Results were tested both at $p<0.001$ uncorrected and at $p<$ 0.05 family-wise error corrected.

RESULTS Clinical, neuropsychological, and structural MRI measures. Table 1 summarizes the main demographic, clinical, and structural MRI measures of patients with pediatric MS and HCs. Compared to HCs, patients with pediatric MS had lower normalized brain volume $(p=0.01)$, gray matter volume $(p=0.02)$ (table 1), and FA values in all tracts analyzed (table e-1).

Thirteen (23\%) patients with pediatric MS were classified as CI. One patient with MS scored $<70$ on the IQ test, and 16 scored in the inferior range $(<90)$. Table 2 summarizes the results of the neuropsychological evaluation in the patients with MS. Thirty-six percent of the CI patients and none of cognitively preserved (CP) patients had impairment on attention tests. Compared to $\mathrm{CP}$ patients, $\mathrm{CI}$ patients with MS had longer disease duration $(p=$ $0.03)$, lower normalized brain volume $(p=0.03)$, lower WM volume $(p=0.01)$, and lower FA values in the tracts connecting the left anterior insula to the anterior cingulate cortex (ACC) $(p=0.01)$ and precuneus $(p=0.04)$ (table e-1).

CCPT fMRI task performance. During fMRI, CCPT performance (percentage of correct and incorrect responses, reaction times) did not differ between patients with pediatric MS and HCs. Compared to
CP patients, CI patients with MS had significantly worse performance (figure e-1).

CCPT task-related activations/deactivations. Table e-2 and figure 1 report brain regions significantly activated/deactivated during the CCPT task load condition in $\mathrm{HCs}$ and patients with pediatric MS. Similar fMRI patterns were detected during the ISI-1, ISI-2, and ISI- 4 conditions but with different $t$ values (data not shown). Both groups showed task-related activations in bilateral precentral cortex, supplementary motor area, inferior parietal lobule, middle temporal gyrus, insula, basal ganglia, and cerebellum, as well as the right inferior frontal gyrus, middle frontal gyrus, and calcarine cortex. Both groups also showed deactivations in bilateral occipital cortex and in regions usually described as part of the default mode network (DMN), bilaterally, including the precuneus, angular gyrus, superior temporal gyrus, middle frontal gyrus (mesial part), and superior frontal gyrus (SFG).

Compared to HCs, patients with pediatric MS showed increased activation of the left thalamus and left anterior insula and decreased deactivation of the left ACC. Compared to HCs, patients with MS also showed increased deactivation of the right precuneus (figure 1).

Effect of cognitive impairment. Table e-3 reports brain regions significantly activated/deactivated during the CCPT task load condition in CP and CI patients with MS separately. Table e-4 and figure 2 show the results of between-group comparisons. 
Table 2 Neuropsychological tests from patients with pediatric MS

All patients with pediatric MS CP patients with pediatric MS $\mathrm{Cl}$ patients with pediatric MS

\begin{tabular}{|c|c|c|c|}
\hline Education, $\mathrm{y}^{\mathrm{a}}$ & $8.7(2.0)$ & $8.6(1.9)$ & $8.8(2.4)$ \\
\hline Global premorbid IQ ${ }^{a}$ & $97.8(18.5)$ & $99.2(18.4)$ & $92.6(19.1)$ \\
\hline$C D I^{\mathrm{a}}$ & $7.9(5.8)$ & $7.6(5.7)$ & $8.6(6.4)$ \\
\hline FSS $^{\mathrm{a}}$ & $27.1(12.1)$ & $27.4(11.6)$ & $26.0(14.2)$ \\
\hline \multicolumn{4}{|l|}{ Memory } \\
\hline SRT-LTS ${ }^{b}$ & $0.08(1.33)$ & $0.38(1.03)$ & $-1.01(1.73)$ \\
\hline SRT-CLTR ${ }^{b}$ & $0.07(1.17)$ & $0.39(0.95)$ & $-1.08(1.18)$ \\
\hline SRT-D $D^{b}$ & $0.02(1.26)$ & $0.30(0.89)$ & $-0.96(1.84)$ \\
\hline SPART ${ }^{\mathrm{b}}$ & $-0.30(1.88)$ & $0.15(1.59)$ & $-1.87(2.05)$ \\
\hline SPART-D & $-0.23(1.44)$ & $0.05(1.19)$ & $-1.19(1.84)$ \\
\hline \multicolumn{4}{|l|}{ Abstract/conceptual reasoning } \\
\hline MCST $^{b}$ & $-0.31(1.39)$ & $-0.16(1.37)$ & $-0.89(1.44)$ \\
\hline \multicolumn{4}{|l|}{ Attention/concentration } \\
\hline SDMT $^{\mathrm{b}}$ & $-0.20(0.99)$ & $-0.07(0.99)$ & $-0.66(0.87)$ \\
\hline TMT-A ${ }^{b}$ & $-0.27(1.18)$ & $0.01(0.94)$ & $-1.26(1.47)$ \\
\hline TMT-B & $-0.40(1.46)$ & $0.00(0.96)$ & $-1.82(2.05)$ \\
\hline \multicolumn{4}{|l|}{ Language } \\
\hline Semantic verbal fluency test ${ }^{b}$ & $-0.25(0.84)$ & $-0.13(0.81)$ & $-0.65(0.86)$ \\
\hline $\begin{array}{l}\text { Phonemic verbal fluency } \\
\text { test }^{\mathrm{b}}\end{array}$ & $-0.24(0.85)$ & $-0.13(0.89)$ & $-0.65(0.58)$ \\
\hline IPT & NA & NA & NA \\
\hline $\mathrm{PCT}^{\mathrm{b}}$ & $-0.58(5.74)$ & $-0.38(6.24)$ & $-1.30(3.61)$ \\
\hline Token test ${ }^{\mathrm{b}}$ & $-0.88(1.46)$ & $-0.86(1.35)$ & $-0.97(1.88)$ \\
\hline ODT & $-0.48(1.31)$ & $-0.27(1.05)$ & $-1.24(1.84)$ \\
\hline
\end{tabular}

Abbreviations: $\mathrm{CDI}=$ Children Depression Inventory; $\mathrm{Cl}=$ cognitively impaired; $\mathrm{CP}=$ cognitively preserved; $\mathrm{FSS}=$ Fatigue Severity Scale; IPT = indication of pictures test; MCST = Modified Card Sorting Test; MS = multiple sclerosis; NA = not applicable; ODT $=$ oral denomination test; $\mathrm{PCT}=$ phrase comprehension test; SDMT $=$ Symbol Digit Modalities Test; SPART = 10/36 Spatial Recall Test; SPART-D = 10/36 Spatial Recall Test Delayed; SRT-CLTR = Selective Reminding Test Consistent Long-Term Retrieval; SRT-D = Selective Reminding Test Delayed; SRT-LTS = Selective Reminding Test Long-Term Storage; TMT-A/B = Trail Making Test A/B.

${ }^{a}$ Mean (SD).

${ }^{\mathrm{b}} \mathrm{z}$ Scores and SD based on a population of pediatric healthy controls matched for age and education. ${ }^{12}$

During the CCPT load condition, compared to HCs, CP patients with MS had increased activation of the left anterior insula and thalamus and decreased deactivation of the ACC and right inferior frontal gyrus. Compared to HCs, CP patients with MS also showed increased deactivation of the right precuneus and superior parietal lobule.

Compared to HCs, CI patients with MS experienced decreased activation of the right postcentral gyrus and increased deactivation of bilateral precuneus. Compared to CI patients, CP patients with pediatric MS had increased recruitment of several areas located mainly in the parietal and occipital lobes and cerebellum (table e-4). They also experienced decreased deactivation of the ACC.

The conjunction analysis identified the left anterior insula and ACC as areas significantly more activated with increasing task difficulty in CP patients with MS compared to the other study groups. Compared to HCs and CP patients, CI patients with MS had lower recruitment of the right postcentral gyrus, right lingual gyrus, right precuneus, left inferior parietal lobule, and left SFG.

Analysis of correlations. In patients with pediatric MS, significant correlations $(p<0.001$ uncorrected $)$ (table 3) were found between increased activation of the left thalamus and lower gray matter volume, higher global cognitive performance $z$ scores, and higher $z$ scores in attentive-executive function domain and between increased deactivation of the right precuneus and higher number of incorrect responses at CCPT and lower FA value of the tract connecting the left anterior insula to ACC. 

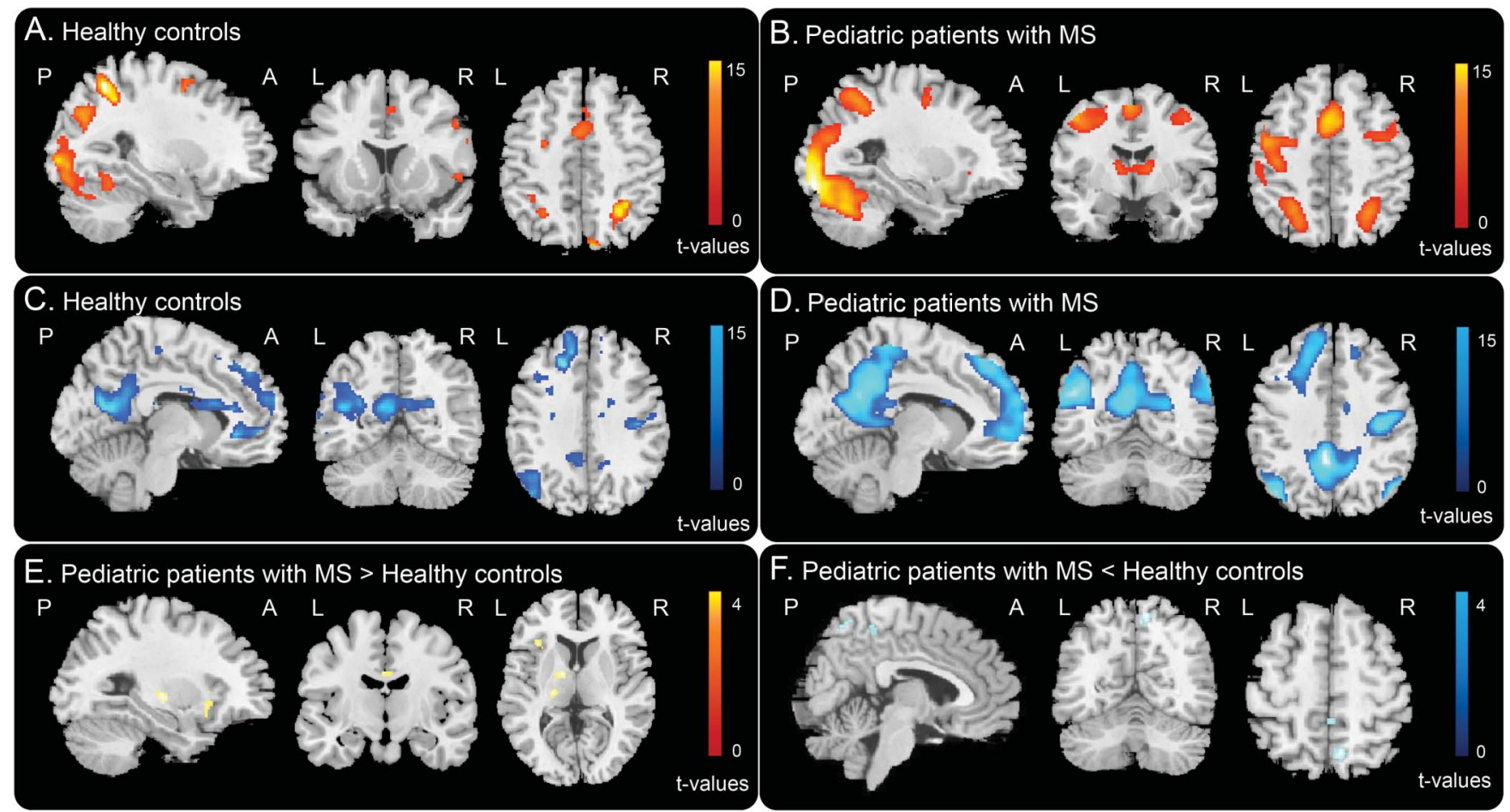

Brain regions showing linearly increasing fMRI activations (A and B) and deactivations (C and D) with increasing Conners Continuous Performance Test (CCPT) difficulty in healthy controls (HCs) (A and C) and pediatric patients with multiple sclerosis (MS) (B and D) (1-sample $t$ tests, $p<0.001$ uncorrected). (E) Areas showing increased activation with increasing CCPT load in patients with pediatric MS vs HCs. (F) Brain areas showing reduced activation. Images are displayed with the neurologic convention. $\mathrm{A}=$ anterior; $\mathrm{P}=$ posterior.

No correlations were found between fMRI findings and $\mathrm{T} 2$ lesion volume and performance of the remaining cognitive domains.

DISCUSSION Here, we applied an active fMRI paradigm to explore the functional competences of the sustained attention network in a relatively large cohort of patients with pediatric MS who underwent a standardized MRI protocol at high magnetic field and a validated neuropsychological assessment. Because cognitive impairment in these patients typically affects multiple domains, its definition is usually based on the number of failed tests at extended neuropsychological batteries. ${ }^{10}$ In line with the literature, ${ }^{10}>30 \%$ of our CI patients with pediatric MS were impaired on attention tests. Although this could represent a limitation for our study, which was focused mainly on sustained attention, it has to be considered that sustained attention is needed for global cognitive functioning ${ }^{1}$ and that only some of its subprocesses are explored by the attention tests that are usually performed. ${ }^{23}$ To explore the functional competence of the attention system in patients with pediatric MS, we investigated the correlations between fMRI findings and CCPT performance during fMRI acquisition and $z$ scores of the attentional domain. Regrettably, we could not obtain neuropsychological data from our sample of pediatric HCs.

Despite the fact that we enrolled only a relatively small number of HCs (thus resulting in limited information on normal variability of fMRI features of typically developing youth), the pattern of functional recruitment of the sustained attention network we found in within-group analyses in pediatric HCs and patients with pediatric MS resembled that described by previous studies in HCs and patients with other neurologic diseases using a similar fMRI paradigm, ${ }^{24,25}$ and it was characterized by a distributed activation of regions located in the frontal, temporal, parietal, and occipital lobes and the cerebellum, all contributing to different aspects of sustained attention. The temporal lobes and parietal cortex mainly integrate polymodal pieces of information involved in exogenous stimuli processing, whereas frontal regions and the cerebellum play a role in reorienting attention $^{26}$ to an exogenous stimulus. We also detected consistent deactivation of regions usually described as part of the $\mathrm{DMN},{ }^{27}$ a network of regions characterized by high activity at rest and low activity during cognitive tasks with focused attention on the external environment. Even if a longitudinal design is needed to confirm our hypothesis, these results suggest the achievement of the age-expected level of functional 

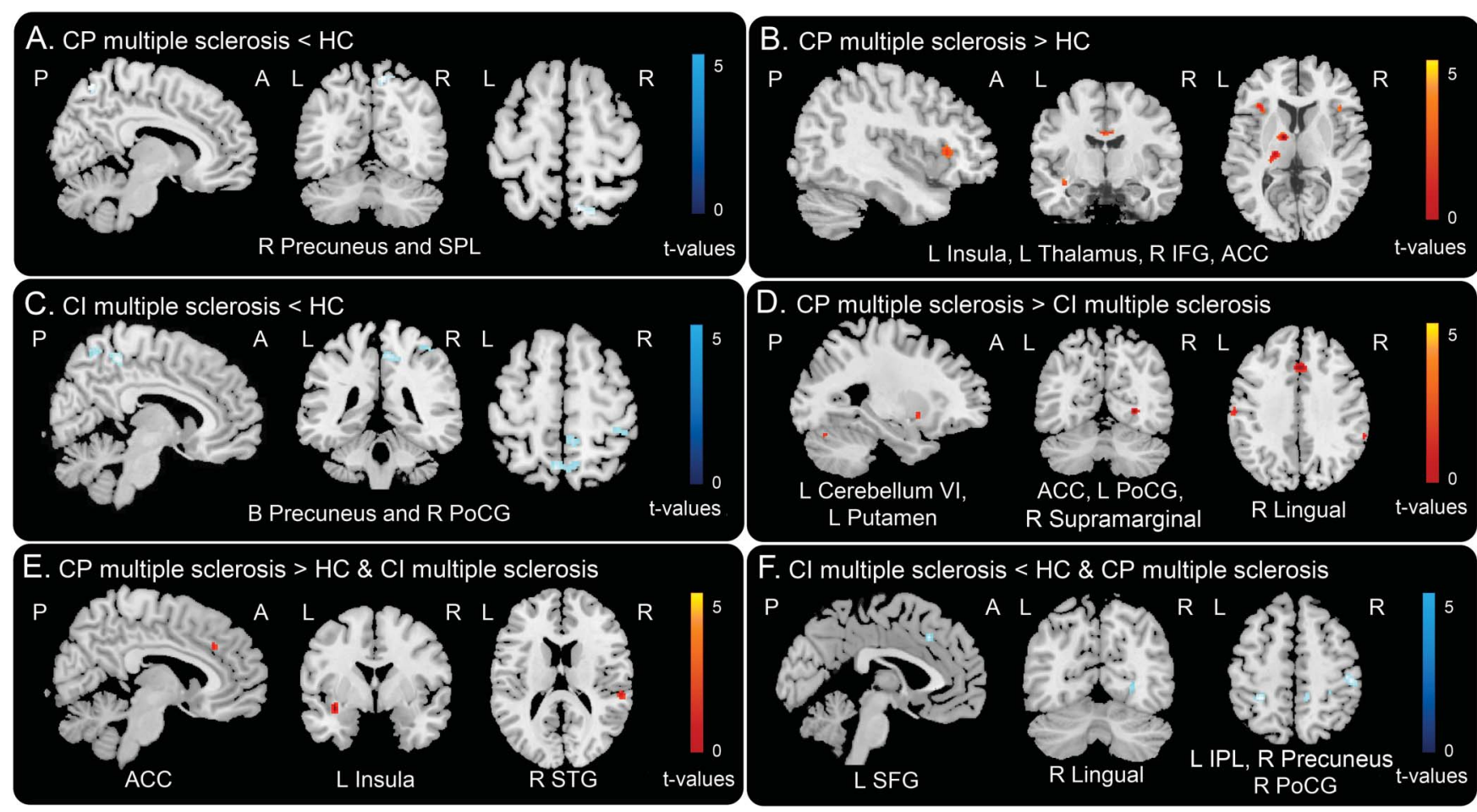

Brain regions showing significantly different $\mathrm{fMRI}$ activations and deactivations with increasing Conners Continuous Performance Test (CCPT) difficulty in cognitively preserved (CP) and cognitively impaired (CI) patients with multiple sclerosis (MS). Results are shown at $p<0.001$ uncorrected. Images are displayed with the neurologic convention. $\mathrm{A}=$ anterior; $\mathrm{ACC}=$ anterior cingulate cortex; $\mathrm{B}=$ bilateral; $\mathrm{HC}=$ healthy control; IFG $=$ inferior frontal gyrus; $\mathrm{IPL}=$ inferior parietal lobule; $\mathrm{MCC}=$ middle cingulate cortex; $\mathrm{P}=$ posterior; $\mathrm{PoCG}=$ postcentral gyrus; $\mathrm{SFG}=$ superior frontal gyrus; $\mathrm{SPL}=$ superior parietal lobule; STG = superior temporal gyrus.

maturation of the network in patients with pediatric MS in terms of ability to activate and deactivate the main regions of the network and topographic representation of these regions.

With increasing CCPT demand, compared to HCs, patients with pediatric MS showed increased activation of the left thalamus, anterior insula, and ACC. They also experienced increased deactivation of the precuneus. Several studies have demonstrated that the basal ganglia, in particular the thalamus, play a crucial role in executive or supervisory mechanisms of attention, including regulation, error monitoring or processing, and sustained vigilance. ${ }^{28,29}$ In adult patients (mean age at evaluation $\approx 19$ years) with disease onset during childhood, a recent study ${ }^{30}$ found that a greater activation of the thalamus in patients compared to HCs during an information processing speed task correlated with better task performance, whereas a resting-state (RS) functional connectivity (FC) study of the DMN found a reduced

\begin{tabular}{|c|c|c|c|c|c|}
\hline \multirow{3}{*}{$\begin{array}{l}\text { Table } 3 \\
\\
\text { Variables }\end{array}$} & \multicolumn{5}{|c|}{$\begin{array}{l}\text { Brain regions showing significant correlations between } \mathrm{fMRI} \text { activations/deactivations during the } \\
\text { CCPT load condition with clinical, neuropsychological, and structural MRI variables in patients with } \\
\text { pediatric MS (multiple regression models adjusted for age and sex; } p<0.001 \text { uncorrected) }\end{array}$} \\
\hline & & CCPT load act & & CCPT load dea & \\
\hline & & Brain regions & $\mathbf{R}$ & Brain regions & $\mathbf{R}$ \\
\hline \multicolumn{2}{|l|}{ GMV } & $L$ thalamus & -0.57 & - & - \\
\hline \multicolumn{4}{|c|}{$\mathrm{L}$ anterior insula/L ACC FA } & $\mathrm{R}$ precuneus & -0.55 \\
\hline \multicolumn{2}{|c|}{ CCPT incorrect responses } & - & - & $\mathrm{R}$ precuneus & -0.53 \\
\hline \multicolumn{2}{|c|}{ Global cognitive z score } & $L$ thalamus & 0.63 & - & - \\
\hline \multicolumn{2}{|c|}{$z$ Score of attentive-executive functions } & $L$ thalamus & 0.49 & - & - \\
\hline
\end{tabular}

Abbreviations: ACC = anterior cingulate cortex; CCPT = Conners Continuous Performance Test; FA = fractional anisotropy; GMV = gray matter volume; MS = multiple sclerosis. 
FC of the thalamus, which was associated with thalamic atrophy. ${ }^{31}$ In our patients with pediatric MS, increased thalamic recruitment during the CCPT task correlated with preserved cognitive performance (particularly with preserved performance in attentiveexecutive functions) and with more pronounced GM atrophy. Combined with the results of the previous studies, ${ }^{30,31}$ these data suggest that thalamic functional abnormalities tend to occur relatively early in the course of the disease as a possible response to disease-related structural damage, in an attempt to preserve cognitive abilities.

Compared to HCs, patients with pediatric MS also had higher recruitment of the anterior insula and ACC. In this case, such activation also helped to distinguish CP patients with MS from the other 2 study groups. The anterior insula and ACC are among the key regions of the salience network, which functions to identify the most relevant among several internal and extrapersonal stimuli to guide behaviour. ${ }^{32}$ Network analysis studies have consistently demonstrated that these 2 regions are involved in switching between brain networks (particularly the executive control and DMN) across task paradigms. ${ }^{33,34}$ On the basis of this finding, a model has been proposed ${ }^{33}$ that posits that the core function of the anterior insula is first to identify stimuli from the vast and continuous stream of sensory stimuli that influence the senses and then to facilitate taskrelated information processing by initiating appropriate transient control signals to engage brain areas mediating attention, working memory, and higherorder cognitive processes, while disengaging the DMN. The ACC is involved in a variety of monitoring, decision making, and cognitive control processes. ${ }^{35}$ Starting from these considerations, the increased recruitment of the insula and ACC detected in CP patients with pediatric MS may represent a key compensatory mechanism for efficient detection of important environmental stimuli and attention shift toward or away from internal cues to maintain adequate performance during a sustained attention task. Supporting this hypothesis, we found higher integrity of the WM tracts connecting the left anterior insula to the ACC and precuneus in $\mathrm{CP}$ compared to $\mathrm{CI}$ patients with MS.

Concomitantly with the presence of areas with increased activation in patients with pediatric MS, we also detected decreased activation of nodal regions of the DMN such as the precuneus and superior parietal lobule, which was due to a higher deactivation in patients compared to controls. This likely reflects a maladaptive mechanism in patients with pediatric MS because it was more pronounced in patients with cognitive impairment and correlated with a poorer performance (higher number of errors) during the
CCPT execution. While CP patients with MS experienced increased deactivation of the right precuneus only, CI patients with MS had a bilateral deactivation of this region. Combined with the previous findings (increased recruitment of the ACC in CP patients with MS), these results suggest that an initial increased deactivation of the precuneus, together with a reduced deactivation of the ACC, as experienced in $\mathrm{CP}$ patients with MS, may represent a compensatory mechanism allowing the patients to maintain an adequate cognitive profile. With disease progression (as reflected by the longer disease duration of CI patients with MS) and accumulation of WM damage (as reflected by decreased FA values in connecting WM tracts in CI patients with MS), a more extended pattern of deactivation (involving the precuneus and SFG) was detected that represents a maladaptive mechanism of cortical reorganization, characterized by an alteration of the shift from the RS condition to sustained attention task performance.

Our results confirm the role of abnormalities of the DMN, in particular of its posterior node centered in the precuneus, in determining cognitive dysfunction in patients with pediatric MS. That the precuneus might be among the first regions affected by the disease in these patients is in line with previous studies, ${ }^{36,37}$ which, by integrating measures derived from structural and fMRI techniques, have demonstrated functional abnormalities at this level, which tended to colocalize with altered structural integrity. Similar to the current findings, a previous RS fMRI study found structural and $\mathrm{AMRI}$ abnormalities of the posterior node of the DMN in CI patients with pediatric MS and increased RS FC of the ACC in CP patients. ${ }^{36}$ A reduced capability to modulate $\mathrm{DMN}$ deactivation with increasing task complexity has also been demonstrated by studies in adult patients with MS. ${ }^{38,39}$

Recent theories, which tend to view the brain as a complex dynamic network, have postulated that abnormalities of interaction over time between the posterior core of the DMN and frontoparietal and subcortical networks, in particular the salience network, might help to explain deficits of cognitive processes after brain damage. ${ }^{20,33,34,40}$ The correlation we found between functional abnormalities of these networks and disruption of structural integrity of critical WM tracts within the networks (in particular the tract connecting the left anterior insula to the ACC) suggests that in patients with pediatric MS, the accumulation of disease-related structural damage might cause a disconnection syndrome resulting in functional and clinical abnormalities. Clearly, longitudinal studies, possibly enrolling very young participants, are now needed to prove such a hypothesis. 


\section{AUTHOR CONTRIBUTIONS}

Ermelinda De Meo contributed to drafting/revising the manuscript and statistical analysis. Lucia Moiola, Angelo Ghezzi, Pierangelo Veggiotti, Ruggero Capra, and Maria Pia Amato contributed to patient enrollment and analysis of the data. Elisabetta Pagani contributed to MRI data postprocessing and statistical analysis. Agnese Fiorino, Lorena Pippolo, and Maria C. Pera contributed to patients' neuropsychological assessment and analysis of the data. Giancarlo Comi contributed to study concept. Andrea Falini contributed to MRI acquisitions and analysis of the data. Massimo Filippi contributed to drafting/revising the manuscript, study concept, and analysis and interpretation of the data. He also acted as study supervisor. Maria A. Rocca contributed to drafting/revising the manuscript and statistical analysis, obtaining funding. All the authors gave their approval to the current version of the manuscript.

\section{STUDY FUNDING}

Partially supported by grants from Italian Ministry of Health (GR-20091529671) and Fondazione Italiana Sclerosi Multipla (FISM-2016-R-23).

\section{DISCLOSURE}

E. De Meo reports no disclosures relevant to the manuscript. L. Moiola received honoraria for speaking or for serving on the advisory board from Sanofi-Genzyme, Biogen, Novartis, and Teva. A. Ghezzi received honoraria for speaking from Biogen-Idec, Merck-Serono, Novartis, Genzyme, Teva, and Allergan; honoraria for consultancy from Merck-Serono, Teva, Novartis, and Biogen-Idec; and support for participation in national and international congresses from Schering, Biogen-Idec, Merck-Serono, Novartis, Genzyme, and Teva. P. Veggiotti reports no disclosures relevant to the manuscript. R. Capra received consulting fees from Novartis and Biogen and lecture fees and/or travel grants from Novartis, Biogen, Genzyme, and Sanofi-Aventis. M. Pia Amato has received research grants and honoraria as a speaker and member of advisory boards from Bayer, Biogen Idec, Merck Serono, Novartis, Sanofi Genzyme, Teva, and Almirall. E. Pagani, A. Fiorino, L. Pippolo, and M. Pera report no disclosures relevant to the manuscript. G. Comi has received compensation for consulting services for Novartis, Teva, Sanofi, Genzyme, Merck, Biogen, Excemed, Roche, Almirall, Chugai, Receptos, and Forward Pharma, as well as compensation for speaking activities from Novartis, Teva, Sanofi, Genzyme, Merck, Biogen, Excemed, and Roche. A. Falini reports no disclosures relevant to the manuscript. M. Filippi is editor-in-chief of the Journal of Neurology; serves on a scientific advisory board for Teva Pharmaceutical Industries; has received compensation for consulting services and/or speaking activities from Biogen Idec, Merk-Serono, Novartis, and Teva Pharmaceutical Industries; and receives research support from Biogen Idec, Teva Pharmaceutical Industries, Novartis, Italian Ministry of Health, Fondazione Italiana Sclerosi Multipla, Cure PSP, Alzheimer's Drug Discovery Foundation, the Jacques and Gloria Gossweiler Foundation (Switzerland), and ARiSLA (Fondazione Italiana di Ricerca per la SLA). M. Rocca received speaker honoraria from Biogen Idec, Novartis, Genzyme, Sanofi-Aventis, Teva, and Merk Serono and receives research support from the Italian Ministry of Health and Fondazione Italiana Sclerosi Multipla. Go to Neurology.org for full disclosures.

Received February 6, 2017. Accepted in final form July 5, 2017.

\section{REFERENCES}

1. Sarter M, Givens B, Bruno JP. The cognitive neuroscience of sustained attention: where top-down meets bottom-up. Brain Res Brain Res Rev 2001;35:146-160.

2. Rubia K, Hyde Z, Halari R, Giampietro V, Smith A. Effects of age and sex on developmental neural networks of visual-spatial attention allocation. Neuroimage 2010; 51:817-827.

3. Travis F. Cortical and cognitive development in 4th, 8th and 12th grade students: the contribution of speed of processing and executive functioning to cognitive development. Biol Psychol 1998;48:37-56.
4. Segalowitz SJ, Santesso DL, Jetha MK. Electrophysiological changes during adolescence: a review. Brain Cogn 2010;72:86-100.

5. Fortenbaugh FC, DeGutis J, Esterman M. Recent theoretical, neural, and clinical advances in sustained attention research. Ann NY Acad Sci 2017;1396:70-91.

6. Smith AB, Halari R, Giampetro V, Brammer M, Rubia K. Developmental effects of reward on sustained attention networks. Neuroimage 2011;56:1693-1704.

7. Aubert-Broche B, Fonov V, Narayanan S, et al. Onset of multiple sclerosis before adulthood leads to failure of ageexpected brain growth. Neurology 2014;83:2140-2146.

8. Rocca MA, Sonkin M, Copetti M, et al. Diffusion tensor magnetic resonance imaging in very early onset pediatric multiple sclerosis. Mult Scler 2016;22:620-627.

9. Shalev L, Kolodny T, Shalev N, Mevorach C. Attention functioning among adolescents with multiple learning, attentional, behavioral, and emotional difficulties. J Learn Disabil 2016;49:582-596.

10. Amato MP, Krupp LB, Charvet LE, Penner I, Till C. Pediatric multiple sclerosis: cognition and mood. Neurology 2016;87:S82-S87.

11. Kurtzke JF. Rating neurologic impairment in multiple sclerosis: an Expanded Disability Status Scale (EDSS). Neurology 1983;33:1444-1452.

12. Amato MP, Goretti B, Ghezzi A, et al. Cognitive and psychosocial features of childhood and juvenile MS. Neurology 2008;70:1891-1897.

13. Portaccio E, Goretti B, Lori S, et al. The Brief Neuropsychological Battery for children: a screening tool for cognitive impairment in childhood and juvenile multiple sclerosis. Mult Scler 2009;15:620-626.

14. Wechsler D. WISC-R Manual for the Wechsler Intelligence Scale for Children-Revised. New York: The Psychological Corporation; 1974.

15. Sepulcre J, Vanotti S, Hernandez R, et al. Cognitive impairment in patients with multiple sclerosis using the Brief Repeatable Battery-Neuropsychology test. Mult Scler 2006;12:187-195.

16. Fuentes A, Collins DL, Garcia-Lorenzo D, et al. Memory performance and normalized regional brain volumes in patients with pediatric-onset multiple sclerosis. J Int Neuropsychol Soc 2012;18:471-480.

17. Homack S, Riccio CA. Conners' Continuous Performance Test (2nd ed.; CCPT-II). J Atten Disord 2006;9:556-558.

18. Chard DT, Jackson JS, Miller DH, Wheeler-Kingshott CA. Reducing the impact of white matter lesions on automated measures of brain gray and white matter volumes. J Magn Reson Imaging 2010;32:223-228.

19. Aron AR, Behrens TE, Smith S, Frank MJ, Poldrack RA. Triangulating a cognitive control network using diffusionweighted magnetic resonance imaging (MRI) and functional MRI. J Neurosci 2007;27:3743-3752.

20. Bonnelle V, Ham TE, Leech R, et al. Salience network integrity predicts default mode network function after traumatic brain injury. Proc Natl Acad Sci USA 2012; 109:4690-4695.

21. Hua K, Zhang J, Wakana S, et al. Tract probability maps in stereotaxic spaces: analyses of white matter anatomy and tract-specific quantification. Neuroimage 2008;39:336347.

22. Friston KJ, Frith CD, Frackowiak RS, Turner R. Characterizing dynamic brain responses with $\mathrm{PMRI}$ : a multivariate approach. Neuroimage 1995;2:166-172. 
23. Egeland J, Kovalik-Gran I. Measuring several aspects of attention in one test: the factor structure of Conners's Continuous Performance Test. J Atten Disord 2010;13: 339-346.

24. Tana MG, Montin E, Cerutti S, Bianchi AM. Exploring cortical attentional system by using fMRI during a Continuous Performance Test. Comput Intell Neurosci 2010:329213.

25. Strazzer S, Rocca MA, Molteni E, et al. Altered recruitment of the attention network is associated with disability and cognitive impairment in pediatric patients with acquired brain injury. Neural Plast 2015;2015:104282.

26. Japee S, Holiday K, Satyshur MD, Mukai I, Ungerleider LG. A role of right middle frontal gyrus in reorienting of attention: a case study. Front Syst Neurosci 2015;9:23.

27. Raichle ME, MacLeod AM, Snyder AZ, Powers WJ, Gusnard DA, Shulman GL. A default mode of brain function. Proc Natl Acad Sci USA 2001;98:676-682.

28. Fan J, Hof PR, Guise KG, Fossella JA, Posner MI. The functional integration of the anterior cingulate cortex during conflict processing. Cereb Cortex 2008;18:796-805.

29. Posner MI, Rothbart MK, Sheese BE, Tang Y. The anterior cingulate gyrus and the mechanism of self-regulation. Cogn Affect Behav Neurosci 2007;7:391-395.

30. Akbar N, Banwell B, Sled JG, et al. Brain activation patterns and cognitive processing speed in patients with pediatric-onset multiple sclerosis. J Clin Exp Neuropsychol 2016;38:393-403.

31. Akbar N, Till C, Sled JG, et al. Altered resting-state functional connectivity in cognitively preserved pediatric-onset
MS patients and relationship to structural damage and cognitive performance. Mult Scler 2016;22:792-800.

32. Seeley WW, Menon V, Schatzberg AF, et al. Dissociable intrinsic connectivity networks for salience processing and executive control. J Neurosci 2007;27:2349-2356.

33. Menon V, Uddin LQ. Saliency, switching, attention and control: a network model of insula function. Brain Struct Funct 2010;214:655-667.

34. Chen T, Cai W, Ryali S, Supekar K, Menon V. Distinct global brain dynamics and spatiotemporal organization of the salience network. PLoS Biol 2016;14:e1002469.

35. Dosenbach NU, Fair DA, Miezin FM, et al. Distinct brain networks for adaptive and stable task control in humans. Proc Natl Acad Sci USA 2007;104:11073-11078.

36. Rocca MA, Absinta M, Amato MP, et al. Posterior brain damage and cognitive impairment in pediatric multiple sclerosis. Neurology 2014;82:1314-1321.

37. Akbar N, Giorgio A, Till C, et al. Alterations in functional and structural connectivity in pediatric-onset multiple sclerosis. PLoS One 2016;11:e0145906.

38. Morgen K, Sammer G, Courtney SM, et al. Distinct mechanisms of altered brain activation in patients with multiple sclerosis. Neuroimage 2007;37:937-946.

39. Rocca MA, Valsasina P, Hulst HE, et al. Functional correlates of cognitive dysfunction in multiple sclerosis: a multicenter fMRI study. Hum Brain Mapp 2014;35: 5799-5814.

40. Leech R, Sharp DJ. The role of the posterior cingulate cortex in cognition and disease. Brain 2014;137:12-32.

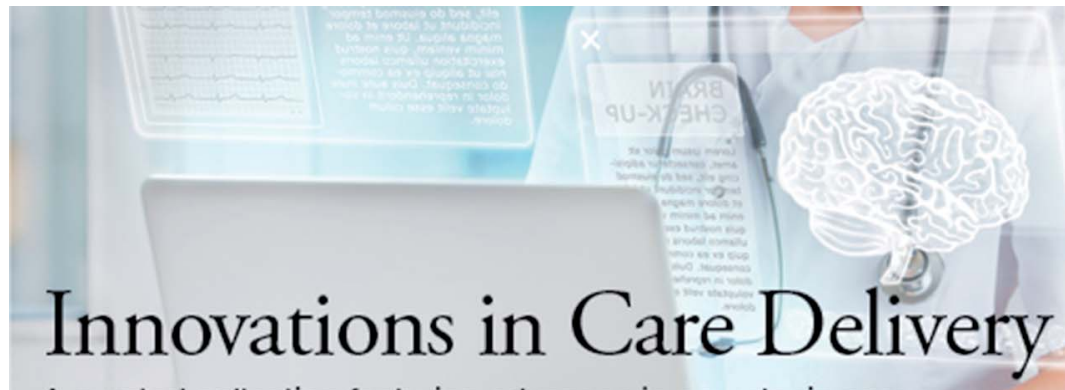

A curated collection featuring advances in neurologic care

\section{NEW! \\ Innovations in Care Delivery - A curated collection featuring advances in neurologic care}

This Neurology ${ }^{\circledR}$ special interest website provides a forum to explore new care models from multiple disciplines, access to sources on health care innovation, and expert opinions on current research from Neurology journals. Curated by Brian C. Callaghan, MD, and Kevin A. Kerber, MD.

Stay ahead of the curve at Neurology.org/innovations. 


\section{Neurology}

\section{MRI substrates of sustained attention system and cognitive impairment in pediatric MS patients}

Ermelinda De Meo, Lucia Moiola, Angelo Ghezzi, et al.

Neurology 2017;89;1265-1273 Published Online before print August 18, 2017

DOI 10.1212/WNL.0000000000004388

This information is current as of August 18, 2017

$\begin{array}{ll}\begin{array}{l}\text { Updated Information \& } \\ \text { Services }\end{array} & \begin{array}{l}\text { including high resolution figures, can be found at: } \\ \text { http://www.neurology.org/content/89/12/1265.full.html }\end{array} \\ \text { Supplementary Material } & \text { Supplementary material can be found at: } \\ & \text { http://www.neurology.org/content/suppl/2017/08/18/WNL.0000000000 } \\ & \text { 004388.DC1 } \\ & \text { This article cites } 38 \text { articles, } 9 \text { of which you can access for free at: } \\ & \text { http://www.neurology.org/content/89/12/1265.full.html\#\#ref-list-1 } \\ \text { References } & \text { This article, along with others on similar topics, appears in the } \\ & \text { following collection(s): } \\ \text { fMRI } & \text { http://www.neurology.org//cgi/collection/fmri } \\ \text { MRI } & \text { http://www.neurology.org//cgi/collection/mri } \\ & \text { Multiple sclerosis } \\ & \text { http://www.neurology.org//cgi/collection/multiple_sclerosis } \\ & \text { Information about reproducing this article in parts (figures,tables) or in } \\ & \text { its entirety can be found online at: } \\ & \text { http://www.neurology.org/misc/about.xhtml\#permissions } \\ \text { Permissions \& Licensing } & \text { Information about ordering reprints can be found online: } \\ & \text { http://www.neurology.org/misc/addir.xhtml\#reprintsus }\end{array}$

Neurology ${ }^{\circledR}$ is the official journal of the American Academy of Neurology. Published continuously since 1951, it is now a weekly with 48 issues per year. Copyright (O 2017 American Academy of Neurology. All rights reserved. Print ISSN: 0028-3878. Online ISSN: 1526-632X.

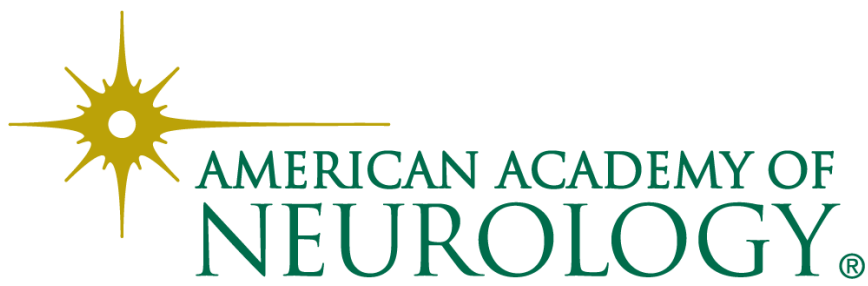

